\title{
Uso do processo de avaliação mútua como suporte ao estudo colaborativo em ambientes de EaD
}

\author{
Anielton José do Nascimento ${ }^{1}$, Clauirton de Albuquerque Siebra ${ }^{1}$ \\ ${ }^{1}$ Departamento de Informática - Universidade Federal da Paraíba (UFPB) \\ João Pessoa - PB - Paraíba \\ anieltonjnegmail.com, clauirton@ci.ufpb.br
}

\begin{abstract}
This work aims to use the mutual evaluation process as a way to assist students in developing their skills through activities and resources that encourage discussion of the content being addressed. This idea is based on works of literature, which indicate that discussion in small groups (4-5 participants are able to improve student learning. Given that there are no studies that show how such discussion may be motivated in distance education, we will address the process of automatic mutual evaluation so that we can identify possible deviations from manual mutual evaluation approach.
\end{abstract}

Resumo. Este trabalho tem o objetivo de utilizar o processo de avaliação mútua como forma de ajudar o aluno a desenvolver suas aptidões através de atividades e recursos que estimulem a discussão do conteúdo que está sendo abordado. Essa ideia é baseada em trabalhos da literatura que indicam que a discussão em grupos reduzidos (de 4 a 5 participantes) é capaz de melhorar a aprendizagem dos alunos. Haja vista que não existem trabalhos que mostrem como tal discussão pode ser motivada na EaD, iremos abordar o processo de avaliação mútua automática de modo que possamos identificar possíveis desvios da abordagem de avaliação mútua manual.

\section{Introdução}

Desde a sua criação, a Internet vem evoluindo a cada dia e hoje podemos afirmar que ela veio renovar, e, muitas vezes, alterar por completo muitos paradigmas da sociedade. Podemos observar também esse fenômeno no surgimento de cursos que exigiam a participação presencial, mas que passaram a ser totalmente online.

A existência de uma diversificada forma de se ofertar cursos online, associada à facilidade de acesso e à flexibilidade de horários, vem contribuir cada vez mais para a popularização dessa modalidade de ensino. Atualmente, encontramos cursos de curta duração, de longa duração, cursos técnicos, cursos de graduação e até de pós-graduação que são oferecidos na modalidade a distância.

Um dos problemas dessa nova tendência no ensino está em como melhorar a capacidade de aprender, sendo o aprendizado cada vez mais distante do professor. Os cursos de EaD trazem um novo tipo de estrutura, em termos de ferramentas e agentes (tutores a distância e presenciais) que auxiliam nessa intermediação entre o professor, o conteúdo e o aluno. Então, o que podemos observar é que nos cursos online surgem novos personagens que intermediam o processo de aprendizagem, com a finalidade de 


\section{CBIE-LACLO 2015}

Anais dos Workshops do IV Congresso Brasileiro de Informática na Educação (CBIE 2015)

auxiliar o professor. $\mathrm{O}$ surgimento desses novos recursos culmina na criação de um ambiente de socialização do conhecimento, fato esse proporcionado pelas discussões em grupos nas ferramentas e/ou atividades de cunho colaborativo.

Para Brindley (2009), os cursos online oferecem a oportunidade de um ambiente de aprendizagem social. Esse fato é verificado pela participação colaborativa dos professores, tutores e demais integrantes para com os alunos. Um fator importante para o autoaprendizado é a interação dos alunos, uma vez que essa interação ajuda no desenvolvimento de habilidades importantes tais como: pensamento crítico, autorreflexão e co-construção do conhecimento.

A interação entre os alunos contribui ainda para a formação de grupos de estudo online, sendo esses uma alternativa para facilitar o processo de aprendizagem dos alunos. De fato, o trabalho em equipe, por diversas vezes, soluciona problemas não detectados através de provas e exercícios. Segundo Blom (2013), por exemplo, a capacidade de pausar uma palestra e discutir seu conteúdo com os seus pares cria oportunidades de aprendizagem.

O nosso interesse, neste trabalho, é melhorar a participação dos alunos em ambientes de Ensino a Distância. Para realizar essa proposta, partimos de um grupo já formado na disciplina, com o objetivo de aplicar estratégias de regulação mútua, de modo a motivar a discussão e a colaboração entre os membros desse grupo.

Para atingir os objetivos desta pesquisa e construir o conhecimento científico, alguns procedimentos metodológicos foram aplicados. Iniciamos o nosso projeto com o estudo do Ambiente Virtual de Avaliação utilizado pela UFPB Virtual e, em seguida, a análise dos dados obtidos para se obter um panorama atual das principais dificuldades encontradas pelos alunos no decorrer do processo de aprendizagem. As conclusões deste estudo foram confrontadas com o conhecimento atual obtido através da revisão da literatura na área.

Após a realização desses dois passos, realizamos a escolha da disciplina com maior taxa de retenção do curso de Licenciatura em Computação da UFPB modalidade a distância. Para avaliarmos e validarmos a nossa metodologia, coletamos as informações e aplicamos a estratégia de regulação mútua na disciplina escolhida.

\section{Fundamentação Teórica}

De acordo com alguns estudiosos como Brindley (2009) e Fardo (2013), vivemos em uma época em que a informação é o maior ativo da sociedade e essa mesma sociedade gera demandas de acesso e processamento cada vez mais rápido da informação, demanda essa que se torna inviável para apenas um indivíduo conseguir realizar sozinho.

Com o advento e popularização dos cursos a distância (online), consegue-se aproximar o conhecimento do aluno. No entanto, muitas vezes, ele não tem com quem debater sobre os assuntos com os quais competem ao meio acadêmico e a sua realidade social. A alternativa para solucionar essa questão é a utilização de processos para facilitar a colaboração sem esquecer de utilizar meios modernos de "medir" essa colaboração, haja vista que muitos saberes permeiam diversas áreas do conhecimento de 


\section{CBIE-LACLO 2015}

Anais dos Workshops do IV Congresso Brasileiro de Informática na Educação (CBIE 2015)

forma que a informação obtida e processada, por diversas vezes, caracteriza-se por ser multi/interdisciplinar.

Para Kop (2011), existem basicamente três desafios para uma aprendizagem colaborativa em cursos a distância. O primeiro desafio está relacionado ao estudo auto direcionado que depende de diversos fatores. Entre eles se destacam a motivação, a iniciativa e a confiança; outros estão relacionados ao controle sobre a atividade de aprendizagem ou a questões de linguagem e comunicação utilizadas nos processos de ensino e aprendizagem. O segundo desafio trata da Presença. Nesse ponto, o autor explica o fato do aluno se sentir acompanhado socialmente pelo professor e pelo tutor. O terceiro ponto citado pelo autor mencionado aborda a questão do aluno ter uma visão/leitura crítica e saber se autoavaliar e avaliar o seu par, principalmente em pequenos grupos de discussão.

Tendo em vista a definição de colaboração apresentada por Kop (2011) como ação e efeito de colaborar, no nosso estudo, utilizamos a colaboração no sentido de compartilhar e debater ideias para se chegar a um objetivo comum que é a aprendizagem. Em seguida, discutimos sobre o processo de avaliação mútua, a maneira como ele é concebido e utilizado.

\subsection{Processo de Avaliação Mútua}

Falar do processo de avaliação mútua não é muito fácil, uma vez que muitos trabalhos utilizam o tema, mas não descrevem diretamente como ele foi aplicado. Na literatura, temos alguns textos pedagógicos como, por exemplo, Perrenoud (2000) que citam a importância do processo de avaliação mútua. Em seu livro intitulado "10 Novas Competências para se Ensinar", Perrenoud cita que uma dessas novas competências é justamente envolver os alunos em suas aprendizagens e em seu trabalho. O trabalho de Perrenoud tem o objetivo de suscitar o desejo de aprender, explicitar a relação com o saber, o sentido do trabalho escolar e desenvolver na criança a capacidade de autoavaliação.

Para Guijarro (2000), o processo de avaliação mútua analisa a forma como os indivíduos reciprocamente regulam a aprendizagem cognitiva de outros processos e envolvem-se em modos comuns de regulação cognitiva. Sob essa ótica, estaríamos trabalhando com indivíduos que sabem o que querem e, participando de um processo de aprendizagem, seriam convidados avaliar a si mesmo e a outros no decorrer do processo de aprendizagem.

De acordo com o que cita Perrenoud (2000), entendemos que o Processo de Avaliação Mútua é importante para o fortalecimento das estruturas educacionais, pois ao inserir o aluno no processo de avaliação esse se sentirá motivado a contribuir para ampliar as relações educacionais existentes. Ao analisarmos o trabalho de Guijarro (2000), concluímos que a autora traz consigo a percepção que o Processo de Avaliação Mútua é importante para a educação, pois ao inserir os alunos em ambientes sociáveis dentro da sala de aula, tais como pequenos grupos de trabalhos, estamos formalizando uma das mais difíceis tarefas educacionais. Para Guijarro (2000) o Processo de Avaliação Mútua corrobora as estruturas educacionais ao inserir os alunos no ambiente de vida na sociedade contemporânea. 


\section{CBIE-LACLO 2015}

Anais dos Workshops do IV Congresso Brasileiro de Informática na Educação (CBIE 2015)

Para Shiba e Sugawara (2014, p. 425), "a percepção de avaliação mútua do trabalho é mais fácil e mais precisa quando é limitada aos membros do mesmo grupo porque os membros do grupo têm testemunhado as atividades dos outros alunos desse grupo". Isso nos leva a concluir que o trabalho em grupos, que se autorregulam (avaliam), tende a produzir melhores resultados, como também cada elemento do grupo tende a melhorar no cumprimento das suas atividades.

Conforme os autores mencionados no parágrafo anterior, a regulação mútua ajuda na percepção do papel do aluno, quais as suas obrigações e o que deve ou não ser feito para aquisição do conhecimento na condição de indivíduo. Segundo Guijarro (2000), a regulação mútua é construída para a utilização de papéis em conjunção uns com os outros. De acordo com o autor, a regulação mútua contribui ainda para o modo como as perguntas são feitas e respondidas, a forma como o conteúdo é clarificado e resumido e a maneira como a aprendizagem é monitorada para a exatidão.

Dentro do grupo, a regulação mútua atua de forma a fazer com que o participante se autoavalie e, consequentemente, obtenha, com maior rapidez, respostas para as suas dúvidas e questionamentos. Dessa forma, o processamento das informações e o acompanhamento do estudo será mais fiel a cada grupo, obedecendo às particularidades e resolvendo problemas como a demora para obter o feedback dos demais participantes. Sob essas condições, entende-se que "o conjunto grupo monitora a sua compreensão em curso em um processo onde os alunos mutuamente regulam a sua aprendizagem conjunta" (GUIJARRO, 2000, p. 43).

De acordo com Shiba e Sugawara (2014), devemos ter cuidado ao utilizar o processo de avaliação mútua haja vista que alguns alunos podem avaliar de forma desonesta, aumentando ou diminuindo a avaliação do seu parceiro. Para diminuir essa possibilidade, nosso trabalho foi proposto de forma que, mesmo que os grupos permaneçam os mesmos do início ao fim de cada disciplina, possa haver um cálculo à parte para validar a percepção dos alunos.

Shiba e Sugawara (2014) propõem, em seu estudo, validar o processo de avaliação mútua através de uma rede de confiança. Com base nessa concepção, nosso estudo pretende validar a confiança das avaliações prestadas pelos participantes dos grupos colaborativos a partir do processo de avaliação mútua automático, utilizando dados que serão coletados e posteriormente calculados nos mesmos moldes da avaliação mútua.

\section{Procedimentos Metodológicos}

A nossa proposta é desenvolver uma alternativa para o fomento da discussão sob os mais diversos temas das mais diversas disciplinas e cursos ministrados a distância. Para isso, partimos da ideia de que pequenos grupos de 4 ou 5 componentes foram formados e, em seguida, utilizamos a estratégia de avaliação/regulamentação mútua.

Dentro da regulamentação mútua, abordamos duas possibilidades na formação do grupo: um que possui liderança e outro em que não existe a figura do líder. Convém salientar que a escolha desse líder será tratada no momento da formação dos grupos e não com os grupos formados. Para a estratégia de grupos com liderança e sem liderança, 
adotamos que, no primeiro caso, o grupo será composto por 5 integrantes. No segundo, como não existe a figura do líder, o grupo será composto por 4 integrantes.

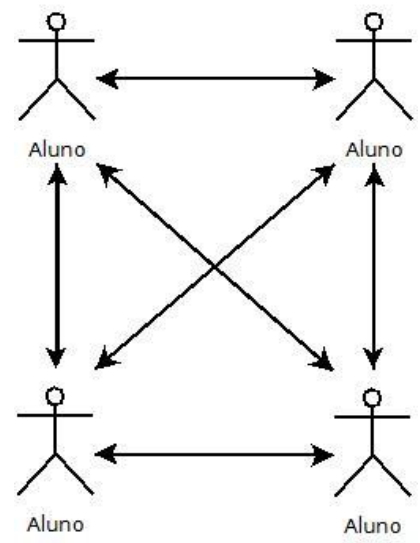

Figura 1. Estratégia do processo de avaliação mútua sem liderança.

No grupo sem o líder, o processo de avaliação mútua é composto por duas notas: a primeira corresponde ao processo de se autoavaliar, já a segunda se refere à média das avaliações recebidas por cada aluno. Como podemos observar na Figura 1.

No grupo que possui um líder, teremos duas formas de avaliação. A primeira será realizada pelo líder que se autoavalia e recebe avaliação dos demais participantes. A segunda será a avaliação dos alunos integrantes do grupo com liderança em que cada aluno se autoavalia e é avaliado pelo líder, formalizando um processo de avaliação mútua em pares, todos com o líder. Na Figura 2, podemos observar o diagrama UML que corresponde ao modelo de avaliação proposto:

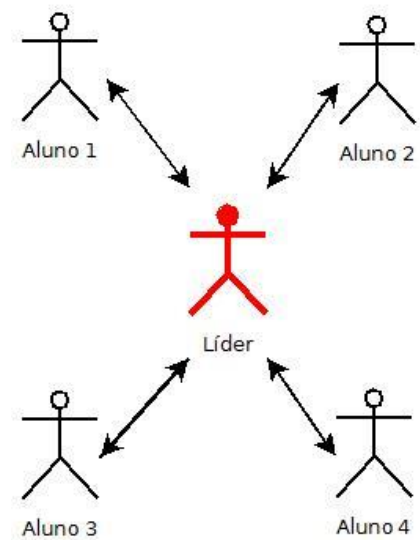

Figura 2. Estratégia do processo de avaliação mútua com liderança.

Nessa ilustração, podemos entender como é realizado o processo de avaliação mútua em grupos utilizando um líder. No outro modelo proposto, onde o grupo não possui um líder, o arranjo do diagrama UML é um pouco diferente, não existindo um elemento central.

Nos dois modelos UML, a seguinte analogia foi utilizada: cada aluno é um ator, cada ator possui uma relação (oculta nos modelos apresentados) que corresponde à ação de se autoavaliar; cada relação apresentada corresponde ao processo mútuo de ser avaliado e de avaliar. 
Para chegar ao processo de avaliação mútua, foram definidas cinco competências a serem avaliadas, sendo elas:

a) Participação na sala de aula: nesse caso, definimos como competência o quão assíduo o aluno é, se ele acessa regularmente a sala de aula, vendo as atividades e participando do curso;

b) Participação nos fóruns e chats: aqui definimos como uma competência o quão o aluno é participativo nos grupos de discussão, fóruns e chats, como ferramentas de fomento a discussão;

c) Contribuição efetiva: acreditamos que essa é uma das mais importantes e mais difíceis competências a serem verificadas. Nessa competência, é verificado o quanto o aluno contribuiu para o desenvolvimento do curso, através das discussões nos fóruns e envio de perguntas.

d) Envio das atividades: nessa competência, avaliamos o quão o aluno é responsável e se ele enviou as atividades obrigatórias e/ou opcionais;

e) Nota obtida nas atividades: se o aluno enviou as atividades, buscamos saber se ele as enviou apenas para cumprir suas obrigações ou se ele efetivamente realizou as atividades.

Para se chegar ao processo de avaliação mútua, foi definido que, em cada uma dessas competências, a avaliação irá obedecer uma escala de 1 a 5 pontos, definida conforme a Tabela 1 a seguir:

Tabela 1. Escala de avaliação de cada competência.

\begin{tabular}{|c|c|}
\hline Escala & Conceito \\
\hline 1 & Péssimo \\
\hline 2 & Ruim \\
\hline 3 & Razoável \\
\hline 4 & Bom \\
\hline 5 & Muito Bom \\
\hline
\end{tabular}

Na Tabela 1, relacionamos os conceitos de avaliação de desempenho de acordo com a escala proposta para o nosso modelo de avaliação mútua. Cada aluno irá se autoavaliar e ser avaliado, de acordo com a proposta do grupo.

A composição do conceito do aluno em cada competência será calculada em duas vertentes, sendo a primeira automaticamente calculada a partir do sistema proposto, que será detalhada em outro momento. A outra vertente dependerá de cada proposta do grupo.

Para os grupos com liderança, os conceitos dos alunos, com exceção do líder, serão calculados a partir da média. Essa média será composta por duas notas: a primeira será obtida através da autoavaliação que cada aluno faz de si e a segunda será a nota dada pelo líder do grupo. A seguir, na (Figura 3), podemos observar como será calculado o conceito do aluno no processo de avaliação mútua com a utilização de liderança: 


\section{CBIE-LACLO 2015}

Anais dos Workshops do IV Congresso Brasileiro de Informática na Educação (CBIE 2015)

$$
\bar{X} C_{z}=\frac{N L C_{Z}+N A A C_{Z}}{2}
$$

Figura 3. Cálculo do conceito para alunos integrantes de grupos com liderança.

Para os grupos sem liderança e para o líder do grupo, o conceito é obtido através de uma média simples em que $50 \%$ é a nota da autoavaliação que cada aluno faz de si, os outros $50 \%$ da nota é obtido a partir da média das notas que o aluno recebeu dos demais colegas. A Figura 4 demonstra como é calculada a nota de cada conceito do aluno que recebe mais de uma avaliação no processo de avaliação mútua:

$$
\bar{X} C_{Z}=N A A C_{Z} * 0,5+\frac{1}{N} \sum_{i=1}^{n} f C_{Z}(i) * 0,5
$$

Figura 4. Cálculo do conceito para alunos que recebem mais de uma avaliação.

Para explicar o processo de avaliação proposto, realizamos, em seguida, a descrição de cada um dos elementos constantes nas duas fórmulas utilizadas.

- $\overline{\mathbf{x}} C_{Z}$ : corresponde à média obtida na competência $z$.

-NLC $_{\mathrm{Z}}$ : corresponde à nota dada pelo líder ao aluno na competência $z$.

-NAAC $\mathbf{Z}_{\mathrm{Z}}$ : corresponde à autoavaliação realizada pelo aluno na competência $z$.

$\bullet \frac{1}{N} \sum_{i=1}^{n} f_{2}(\mathbf{i})$ : corresponde ao cálculo da média aritmética de $n$ conceitos recebidos pelo aluno na competência $z$.

De acordo com o sistema proposto, o processo de avaliação mútua define uma pontuação do aluno para cada competência avaliada e essa pontuação será confrontada com a pontuação que internamente o sistema irá calcular. Esse cálculo automático obedece aos mesmos critérios das competências elencadas que serão comparados com todos os alunos da turma. Ao final, a pontuação do aluno em cada competência será uma média ponderada entre a pontuação obtida no processo de avaliação mútua e a nota calculada pelo sistema. Compete ainda informar que a ponderação será definida pelo tutor/professor de cada disciplina, obedecendo uma escala de 1 a 5 conforme a Tabela 2:

Tabela 2. Escala de ponderação utilizada.

\begin{tabular}{|c|c|}
\hline Escala & Ponderação Utilizada \\
\hline 1 & $90 \%$ PAM e 10\% PAMA \\
\hline 2 & $75 \%$ PAM e $25 \%$ PAMA \\
\hline 3 & $50 \%$ PAM e 50\% PAMA \\
\hline 4 & $25 \%$ PAM e $75 \%$ PAMA \\
\hline 5 & $10 \%$ PAM e $90 \%$ PAMA \\
\hline
\end{tabular}

Na Tabela 2, apresentada acima, adotamos a seguinte nomenclatura:

PAM - Processo de Avaliação Mútua;

PAMA - Processo de Avaliação Mútua Automático.

Nesse ponto, definimos uma certa flexibilidade no modelo proposto de avaliação. Entendemos que dessa forma flexível é possível atenuar possíveis diferenças 
CBIE-LACLO 2015

Anais dos Workshops do IV Congresso Brasileiro de Informática na Educação (CBIE 2015)

entre o que o Processo de Avaliação Mútua e o Processo de Avaliação Mútua Automático conseguiram obter de cada aluno.

\section{Estudo de Caso: Aplicando a Avaliação Mútua Automático}

Para realizarmos o processo de avaliação mútua automático, foi necessário responder às perguntas das competências elencadas. Nesse processo, buscamos também distinguir cada ação realizada pelo aluno. Ao realizamos a consulta ao banco de dados, obtivemos, para cada aluno, um arquivo com os seus respectivos acessos.

$\mathrm{Na}$ consulta realizada, os dados foram separados e tabulados de tal forma a obter a informação necessária para responder aos questionamentos das competências. Esses resultados obtidos correspondem aos dados de participação dos alunos de acordo com a atividade.

A partir da tabulação dos dados referenciados e da obtenção da informação de acesso dos alunos, podemos realizar o cálculo de algumas medidas de desempenho. Na Tabela 3, podemos observar as principais medidas de desempenho. Alguns dados nos chamam a atenção, como é o caso do alto índice do Coeficiente de Variação da atividade do tipo chat, além do alto grau da Variância de todos os acessos dos alunos.

Tabela 3. Relação de acesso dos alunos por medidas de desempenho.

\begin{tabular}{|l|r|r|r|r|r|}
\hline \multirow{2}{*}{ Medidas de Desempenho } & \multirow{2}{*}{$\begin{array}{c}\text { Todos } \\
\text { Acessos }\end{array}$} & \multicolumn{4}{|c|}{ Acesso por Atividade } \\
\cline { 3 - 6 } & Arquivo & Fórum & Tarefa & \multicolumn{1}{c|}{ Chat } \\
\hline Variância & $296.005,70$ & 333,27 & $21.159,41$ & $6.704,00$ & 287,41 \\
\hline Desvio-padrão & 544,06 & 18,26 & 145,46 & 81,88 & 16,95 \\
\hline Coeficiente de Variação & 50,73 & 56,83 & 50,93 & 41,14 & 137,00 \\
\hline
\end{tabular}

De posse dos conceitos obtidos de cada aluno, foram realizados alguns testes com a finalidade de obter resultados sobre o modelo proposto. Após todos os cálculos realizados, chegamos a Tabela 4 que contém todos os conceitos dos alunos em suas respectivas competências.

Tabela 4. Alunos por Competência.

\begin{tabular}{|l|l|l|l|l|l|}
\hline \multirow{2}{*}{ Alunos } & \multicolumn{5}{|c|}{ Conceito na Competência } \\
\cline { 2 - 6 } & $\mathbf{1}$ & $\mathbf{2}$ & $\mathbf{3}$ & $\mathbf{4}$ & $\mathbf{5}$ \\
\hline Aluno 01 & 3 & 5 & 3 & 5 & 4 \\
\hline Aluno 02 & 5 & 3 & 5 & 4 & 5 \\
\hline Aluno 03 & 1 & 1 & 1 & 1 & 1 \\
\hline Aluno 04 & 5 & 5 & 3 & 4 & 2 \\
\hline Aluno 05 & 5 & 5 & 1 & 5 & 2 \\
\hline Aluno 06 & 1 & 1 & 1 & 1 & 1 \\
\hline Aluno 07 & 1 & 1 & 2 & 2 & 4 \\
\hline Aluno 08 & 4 & 5 & 2 & 4 & 5 \\
\hline
\end{tabular}

Com as informações acima, podemos já detectar que dois alunos (Aluno 03 e Aluno 06) obtiveram um desempenho muito abaixo do esperado em todas as competências. Tal desempenho culminou consequentemente na reprovação direta por média dos alunos em questão. Da mesma forma, podemos observar que dois alunos (02 e 08) se destacaram de forma positiva, obtendo conceitos acima do esperado nas 
competências. O fato de existir dois alunos com conceitos em todas as competências abaixo do esperado, em contraste com outros dois alunos com conceitos elevados, culminou na obtenção de altos índices de variância em cada competência, conforme podemos observar na Tabela 5 abaixo:

Tabela 5. Medidas de Desempenho da Turma.

\begin{tabular}{|l|r|r|r|r|r|}
\hline \multirow{2}{*}{ Medidas de Desempenho } & \multicolumn{5}{|c|}{ Competências } \\
\cline { 2 - 6 } & \multicolumn{1}{|c|}{$\mathbf{1}$} & \multicolumn{1}{c|}{$\mathbf{3}$} & \multicolumn{1}{c|}{$\mathbf{4}$} & \multicolumn{1}{c|}{$\mathbf{5}$} \\
\hline Média & 3,13 & 3,25 & 2,25 & 3,25 & 3,00 \\
\hline Variância & 3,554 & 3,929 & 1,929 & 2,786 & 2,857 \\
\hline Desvio-padrão & 1,885 & 1,982 & 1,389 & 1,669 & 1,690 \\
\hline Coeficiente de Variação & 60,323 & 60,987 & 61,721 & 51,355 & 56,344 \\
\hline Erro padrão da média & 0,666 & 0,701 & 0,491 & 0,590 & 0,598 \\
\hline
\end{tabular}

Nos chama a atenção o Coeficiente de Variação das três primeiras competências, estando todos acima de 60 . Como as três primeiras competências avaliam a participação do aluno na disciplina, podemos concluir que a falta de interação dos alunos pode ter sido um fator de reprovação deles.

\section{Conclusões e Trabalhos Futuros}

A maior contribuição do processo de avaliação mútua é para o aluno, uma vez que ele pode verificar e comparar o seu desempenho com o grupo ao qual ele pertence e com o da turma. Dessa forma, ele poderá se manter motivado a participar de todas as atividades. De acordo com o que foi estudado, conseguimos identificar que a interação e participação dos alunos nos fóruns e chats é determinante para a obtenção de êxito nas disciplinas ministradas a distância.

A proposta dessa pesquisa é apresentar o processo de avaliação mútua para motivar a participação e consequentemente o desempenho dos alunos nos cursos ofertados na modalidade a distância. No decorrer da pesquisa e após a revisão da literatura, entendemos que o processo de avaliação mútua precisava ser validado e, para isso, desenvolvemos esse método de avaliação automatizado, utilizando as mesmas métricas do processo de avaliação mútua. O próximo passo dessa pesquisa será aplicar o processo de avaliação mútua com os alunos e comparar os resultados com o processo de avaliação mútua automático.

\section{Referências}

BLOM, Jan et al. 2013. "MOOCs are More Social than You Believe" In: eLearning Papers, n. 3.

BRINDLEY, Jane E.; WALTI, Christine; BLASCHKE, Lisa M. 2009. "Creating Effective Collaborative Learning Groups" In: Online Environment. International Review Of Research In Open \& Distance Learning, v. 10, n. 3.

Censo EAD.BR: 2013. "Relatório Analítico da Aprendizagem a Distância no Brasil 2012” = Censo EAD.BR: Analytic Report of Distance Learning in Brazil/[traduzido por Opportunity Translations]. - Curitiba: Ibpex. 
FARDO, Marcelo Luís. 2013. “A gamificação como estratégia pedagógica: estudo de elementos dos games aplicados em processos de ensino e aprendizagem". Dissertação (Mestrado). Universidade de Caxias do Sul, Programa de Pós-Graduação em Educação.

GUIJARRO, Rosa Blanco. 2000. "Inclusive education in Latin America". In: Meeting special and diverse educational needs: Meeting special education a reality. Helsinki, Finland: Ministry of Foreign Affairs, p. 40-51.

KOP, Rita. 2011. "The challenges to connectivist learning on open online networks: Learning experiences during a massive open online course". In: The International Review of Research in Open and Distance Learning, Special Issue-Connectivism: Design and Delivery of Social Networked Learning, v. 12, n. 3, p. 19-38.

PERRENOUD, Philippe. 2000. "10 novas competências para ensinar". Artmed editor.

SHIBA, Yumeno; SUGAWARA, Toshiharu. 2014. "Fair assessment of group work by mútual evaluation based on trust network". In: Frontiers in Education Conference (FIE), 2014 IEEE. IEEE, p. 1-7. 